\title{
FACTORES DE RIESGO DETERMINANTES EN LA AMPUTACIÓN DE PACIENTES CON PIE DIABÉTICO EN EL SERVICIO DE MEDICINA DEL HOSPITAL LUIS N. SAENZ EN EL PERÍODO, ENERO 2015 - JULIO 2016
}

\author{
DETERMINING RISK FACTORS IN THE AMPUTATION OF PATIENTS WITH DIABETIC \\ FOOT IN THE MEDICINE SERVICE OF THE HOSPITAL LUIS N. SAENZ IN THE PERIOD, \\ JANUARY 2015 TO JULY 2016 \\ Lidia Fiorella Nicho-Alegre ${ }^{1, a}$, Consuelo Luna-Muñoz ${ }^{1,2, a, b, c}$, Jhony A. De La Cruz -Vargas²,de,f
}

\begin{abstract}
RESUMEN
Objetivos: Identificar los factores de riesgo determinantes para la amputación de pacientes con pie diabético en el servicio de Medicina del Hospital Luis N. Sáenz en el período Enero 2015 - Julio 2016. Métodos: Estudio observacional, retrospectivo, analítico tipo casos y controles donde se incluyó 219 historias clínicas de pacientes con diabetes mellitus tipo 2 con complicaciones tardías: Pie diabético, distribuidos en: un grupo caso (108 pacientes con pie diabético amputado) y otro grupo control (108 pacientes con pie diabético no amputado). Se realizó la recolección de datos en el programa Microsoft Excel y el análisis estadístico mediante el programa SPSS v.23. Resultados: En el análisis sobre edad, se encontró que la edad de mayor/igual a 75 años es un factor de riesgo para la amputación $(\mathrm{OR}=3,808 ; \mathrm{IC}=2,103-6,897)$. En cuanto al sexo se encontró que el sexo masculino es un factor de riesgo para la amputación $(O R=2,460 ; \mathrm{IC}=1,217-4,972)$. En general en relación a los factores de riesgo para amputación se obtuvo: el tiempo de evolución de la enfermedad mayor/igual a 10 años un (OR=3,206; IC=95\% 1,578-6,514), nivel de glicemia mayor/igual a $110 \mathrm{mg} / \mathrm{dl}$ un $(\mathrm{OR}=3,683 ; \mathrm{IC}=95 \%$ 2,055-6,602) y el intervalo de amputación según la escala de Wagner mayor/igual a III tuvo un mayor $\mathrm{OR=5,624}(\mathrm{IC}=3,076$ 10,283). Conclusión: Los factores de riesgo determinantes para la amputación de pacientes con pie diabético hospitalizados en el Hospital Luis N. Sáenz PNP fueron: la edad mayor/igual a 75 años, el sexo masculino, el tiempo de evolución de la enfermedad mayor/igual a 10 años, el nivel de glicemia mayor/igual a $110 \mathrm{mg} / \mathrm{dl}$ y el grado de lesión según la escala de Wagner mayor/igual a III.
\end{abstract}

Palabras clave: Diabetes mellitus; Pie diabético; Factores de riesgo; Amputación. (fuente: DeCS BIREME)

\begin{abstract}
Objective: To identify the determinant risk factors for the amputation of patients with diabetic foot in the service of Medicine of the Hospital Luis N. Sáenz in the period January 2015 - July 2016. Methods: An observational, retrospective, case-control and analytical study of 219 clinical histories of patients with type 2 diabetes mellitus with late complications: Diabetic foot, distributed in: one case group (108 patients with amputated diabetic foot) and another control group (108 patients with non-amputated diabetic foot). Data collection was performed in the Microsoft Excel program and statistical analysis using the SPSS v. 23 program. Results: In the analysis on age, it was found that the age of greater/equal to 75 years is a risk factor for amputation ( $O R=3.808 ; \mathrm{IC}=2.103-6.897)$. Male sex was a risk factor for amputation ( $O R=2,460 ; \mathrm{IC}=1,217-4,972)$. In general, regarding the risk factors for amputation, the time of evolution of the disease was greater than or equal to 10 years $(\mathrm{OR}=3.206 ; \mathrm{IC}=95 \% 1,578-6,514)$, blood glucose level greater than or equal to $110(\mathrm{OR}=3.683$, $\mathrm{IC}=95 \%$ 2.055-6.602) and the amputation interval according to the Wagner scale greater than or equal to III had a greater $\mathrm{OR}=5.624(\mathrm{IC}=3.076-10.283)$. Conclusions: The determinant risk factors for the amputation of diabetic foot patients hospitalized at the Luis N. Sáenz PNP Hospital were: age greater than or equal to 75 years, male, time of disease evolution greater than or equal to 10 years, Blood glucose level greater than or equal to $110 \mathrm{mg} / \mathrm{dl}$ and degree of injury according to the Wagner scale greater than or equal to III.
\end{abstract}

Key words: Diabetes mellitus; Diabetic foot; Risk factors; Amputation. (source: MeSH NLM)

\footnotetext{
1 Internado Médico , Hospital Luis N. Saenz, Lima-Perú.

${ }^{2}$ Instituto de Investigación en Ciencias Biomédicas. Facultad de Medicina Humana. Universidad Ricardo Palma. Lima. Perú.

a Médico Cirujano, b Doctor en Salud Pública, c Especialista en Pediatría, d Médico Cirujano, e Especialista en Oncologia Medica,

Magister en Investigación Clínica y Doctor en Medicina.

Correspondencia: Lidia Fiorella Nicho Alegre. Celular: 949292239. Correo: lifinial22@hotmail.com
}

Citar como: Lidia Fiorella Nicho-Alegre, Consuelo Luna-Muñoz. Factores de riesgo determinantes en la amputación de pacientes con pie diabético en el servicio de medicina del Hospital Luis N. Saenz en el período, enero 2015-julio 2016. [Artículo Original]. Rev. Fac. Med. Hum. 2017;17(1):72-78. DOI 10.25176/RFMH.v17.n1.751 


\section{INTRODUCCIÓN}

La diabetes es una enfermedad crónica que aparece cuando el páncreas no produce insulina suficiente o cuando el organismo no utiliza eficazmente la insulina que produce, como sabemos la insulina es una hormona que regula el azúcar en la sangre. El efecto de la diabetes no controlada es la hiperglicemia que con el tiempo daña gravemente muchos órganos y sistemas, especialmente los nervios y los vasos sanguíneos; ocurriendo así consecuencias frecuentes como la neuropatía de los pies combinada con la reducción de flujo sanguíneo incrementando el riesgo de úlceras de los pies, infección y en última instancia la amputación.

Una de las complicaciones que se destaca es el pie diabético que evoluciona progresivamente presentando: infección, ulceración y destrucción profunda de los tejidos asociados a problemas neurológicos y de vasculopatía periférica, daño articular, dermatológico y daño de tejidos blandos. Es por ello considerado un problema médico, social y económico en todo el mundo pues es una de las complicaciones más costosas de la diabetes. La gran mayoría de pacientes que presentan pie diabético suelen complicarse con úlceras que obligan a la amputación menor o mayor del miembro inferior.

El examen y los cuidados frecuentes de los pies pueden ayudar a evitar la amputación además de un buen control de los factores de riesgo del pie diabético desempeñando así un papel fundamental en la reducción de la incidencia de amputaciones. Es importante aclarar que la neuropatía diabética y la enfermedad arterial periférica contribuyen al incremento de la morbilidad y la mortalidad por pie diabético por lo que la identificación temprana de estos eventos por medio de la anamnesis, el examen físico y las recomendaciones de cuidado son actividades preventivas.

Si no se cuida la aparición de úlceras en el pie con frecuencia conllevaran a la pérdida de la viabilidad del miembro inferior y por consiguiente la amputación, deterioro funcional, psíquico y económico; hecho que genera un gran impacto económico en el sistema de salud constituyendo así un problema de salud pública que influye en la calidad de vida de las personas afectadas y sus familias.

Por lo tanto las complicaciones devastadoras del pie diabético pueden ser prevenidas en la mayoría de los casos con diagnóstico precoz y educación, dado que representan una estrategia costo-efectiva en la disminución del riesgo de amputaciones en los pacientes diabéticos con complicaciones tardías como lo es el pie diabético. La OMS calcula que el tratamiento y la atención básica de la diabetes permitirían prevenir hasta el $80 \%$ de las amputaciones de pies diabéticos.

\section{MÉTODOS}

Estudio Observacional, retrospectivo, analítico tipo casos y controles donde se incluyó 218 historias clínicas de pacientes con pie diabético, distribuidos en: Grupo caso (109 pacientes con pie diabético amputados) y Grupo control (109 pacientes con pie diabético no amputados). Se realizó la recolección de datos en el programa Microsoft Excel y el análisis estadístico mediante el programa SPSS v.23. El tamaño de la muestra fue calculado mediante la fórmula de casos y controles con un nivel de confianza de $95 \%$, con un porcentaje de error de $5 \%$ en un tamaño poblacional de 500 pacientes y una distribución de las respuestas de 50\%. La técnica de recolección de datos se realizó a partir de historias clínicas, por lo que se elaboró una ficha de recolección de datos donde se encontraban las variables: Pie diabético, edad, sexo, tiempo de evolución de enfermedad, nivel de glicemia y grado de lesión según escala de Wagner. Se procedió al análisis estadístico en el programa SPSS v.23, para el que se utilizó la prueba $x^{2}$ para determinar la asociación, se analizó además el Odds Ratio (OR) con intervalos de confianza al 95\% (IC-95\%) para determinar el riesgo. Considerando significativo todo valor de $p<0.05$. Los aspectos éticos considerados en este trabajo de investigación guardo confidencialidad de sus datos, además se contó con la aprobación del comité de ética en investigación de la Facultad de Medicina de la Universidad Ricardo Palma.

\section{RESULTADOS}

Al culminar con la recolección de datos, estos datos fueron procesados y presentados en tablas y/o gráficos estadísticos para realizar su análisis e interpretación considerando los objetivos y la operacionalizacion de variables, como se detalla a continuación: 
Tabla 1. Edad como factor de riesgo determinante para amputación por pie diabético en pacientes hospitalizados en el Servicio de Medicina del Hospital Luis N. Sáenz periodo Enero 2015-Julio 2016.

\begin{tabular}{|c|c|c|c|c|c|c|c|c|}
\hline \multirow{3}{*}{ Edad } & \multicolumn{4}{|c|}{ AMPUTACIÓN POR PIE DIABÉTICO } & \multirow{3}{*}{ P* } & \multirow{3}{*}{ OR** } & \multirow{2}{*}{\multicolumn{2}{|c|}{ IC AL $95 \%$}} \\
\hline & \multicolumn{2}{|c|}{ Si } & \multicolumn{2}{|c|}{ No } & & & & \\
\hline & $\mathbf{N}^{\circ}$ & $\%$ & $\mathbf{N}^{\circ}$ & $\%$ & & & Inferior & Superior \\
\hline$>=75$ años & 86 & $78.9 \%$ & 54 & $49.5 \%$ & & & & \\
\hline $\begin{array}{c}\text { 60-74 } \\
\text { años }\end{array}$ & 23 & $21.1 \%$ & 55 & $50.5 \%$ & 0,001 & 3,808 & 2,103 & 6,897 \\
\hline Total & 109 & $100.0 \%$ & 109 & $100.0 \%$ & & & & \\
\hline
\end{tabular}

Fuente: INICIB-FAMURP/ HOSPITAL LUIS N. SAENZ

En referencia a la tabla 1, se observa que la edad mayor o igual a 75 años de los pacientes con pie diabético se relaciona significativamente con la amputación $(p=0,001)$, siendo un factor de riesgo para la amputación $(\mathrm{OR}=3,808$; IC=2,103-6,897); es decir el riesgo de que un paciente con pie diabético sea amputado es 3,8 veces mayor en un paciente con edad mayor o igual a 75 años respecto a un paciente con edad entre 60 y 74 años.

Tabla 2 . Sexo como factor de riesgo determinante para amputación por pie diabético en pacientes hospitalizados en el Servicio de Medicina del Hospital Luis N. Sáenz periodo Enero 2015-Julio 2016.

\begin{tabular}{|c|c|c|c|c|c|c|c|c|}
\hline \multirow{3}{*}{ Sexo } & \multicolumn{4}{|c|}{ AMPUTACIÓN POR PIE DIABÉTICO } & \multirow{3}{*}{ P* } & \multirow{3}{*}{$\mathbf{O R}^{* *}$} & \multirow{2}{*}{\multicolumn{2}{|c|}{ IC AL $95 \%$}} \\
\hline & & & & & & & & \\
\hline & $\mathbf{N}^{\circ}$ & $\%$ & $\mathbf{N}^{\circ}$ & $\%$ & & & Inferior & Superior \\
\hline Masculino & 95 & $87.2 \%$ & 80 & $73.4 \%$ & & & & \\
\hline Femenino & 14 & $12.8 \%$ & 29 & $26.6 \%$ & 0,011 & 2,460 & 1,217 & 4,972 \\
\hline Total & 109 & $100.0 \%$ & 109 & $100.0 \%$ & & & & \\
\hline
\end{tabular}

Fuente: INICIB-FAMURP/ HOSPITAL LUIS N. SAENZ

Con respecto a la tabla 2 se encontró que en los pacientes de sexo masculino la amputación por pie diabético representó un $87.2 \%$, relacionándose significativamente con la amputación por pie diabético $(p=0,011)$. Existe factor de riesgo para amputación por pie diabético $(\mathrm{OR}=2,460 ; \mathrm{IC}=1,217-4,972)$; lo cual quiere decir que el riesgo de que un paciente de sexo masculino sea amputado por pie diabético es 2,46 veces mayor que las pacientes de sexo femenino.

Tabla 3 . Sexo como factor de riesgo determinante para amputación por pie diabético en pacientes hospitalizados en el Servicio de Medicina del Hospital Luis N. Sáenz periodo Enero 2015-Julio 2016.

\begin{tabular}{|c|c|c|c|c|c|c|c|c|}
\hline \multirow{3}{*}{$\begin{array}{l}\text { TIEMPO DE } \\
\text { DIABETES } \\
\text { MILLETUS }\end{array}$} & \multicolumn{4}{|c|}{ AMPUTACIÓN POR PIE DIABÉTICO } & \multirow{3}{*}{ P* } & \multirow{3}{*}{$\mathbf{O R}^{* *}$} & \multirow{2}{*}{\multicolumn{2}{|c|}{ IC AL $95 \%$}} \\
\hline & \multicolumn{2}{|c|}{ Si } & \multicolumn{2}{|c|}{ No } & & & & \\
\hline & $\mathbf{N}^{\circ}$ & $\%$ & $\mathbf{N}^{\circ}$ & $\%$ & & & Inferior & Superior \\
\hline$>=10$ años & 96 & $88.1 \%$ & 76 & $69.7 \%$ & & & & \\
\hline$<10$ años & 13 & $11.9 \%$ & 33 & $30.3 \%$ & 0,001 & 3,206 & 1,578 & 6,514 \\
\hline Total & 109 & $100.0 \%$ & 109 & $100.0 \%$ & & & & \\
\hline
\end{tabular}

Fuente: INICIB-FAMURP/ HOSPITAL LUIS N. SAENZ 
Referente al tiempo de evolución de la Diabetes Mellitus (mayor/igual a 10 años y menor a 10 años), la tabla 3 muestra que el $88.1 \%$ de pacientes con pie diabético que tuvieron la enfermedad más de 10 años fueron amputados teniendo mayor proporción en comparación con los pacientes que tuvieron tiempo de enfermedad menor de 10 años con un 11.9\%; observándose que el tiempo de enfermedad de diabetes mellitus mayor igual a 10 años se relaciona significativamente con la amputación por pie diabético $(p=0.001)$, siendo un factor de riesgo $(O R=3,206$; $I C=95 \%$ 1,578-6,514), es decir, que el riesgo que un paciente sea amputado es 3,206 veces mayor que en quienes presentan la enfermedad por menos de 10 años.

Tabla 4 . Nivel de glicemia como factor de riesgo determinante para amputación por pie diabético en pacientes hospitalizados en el Servicio de Medicina del Hospital Luis N. Sáenz periodo Enero2015-Julio 2016.

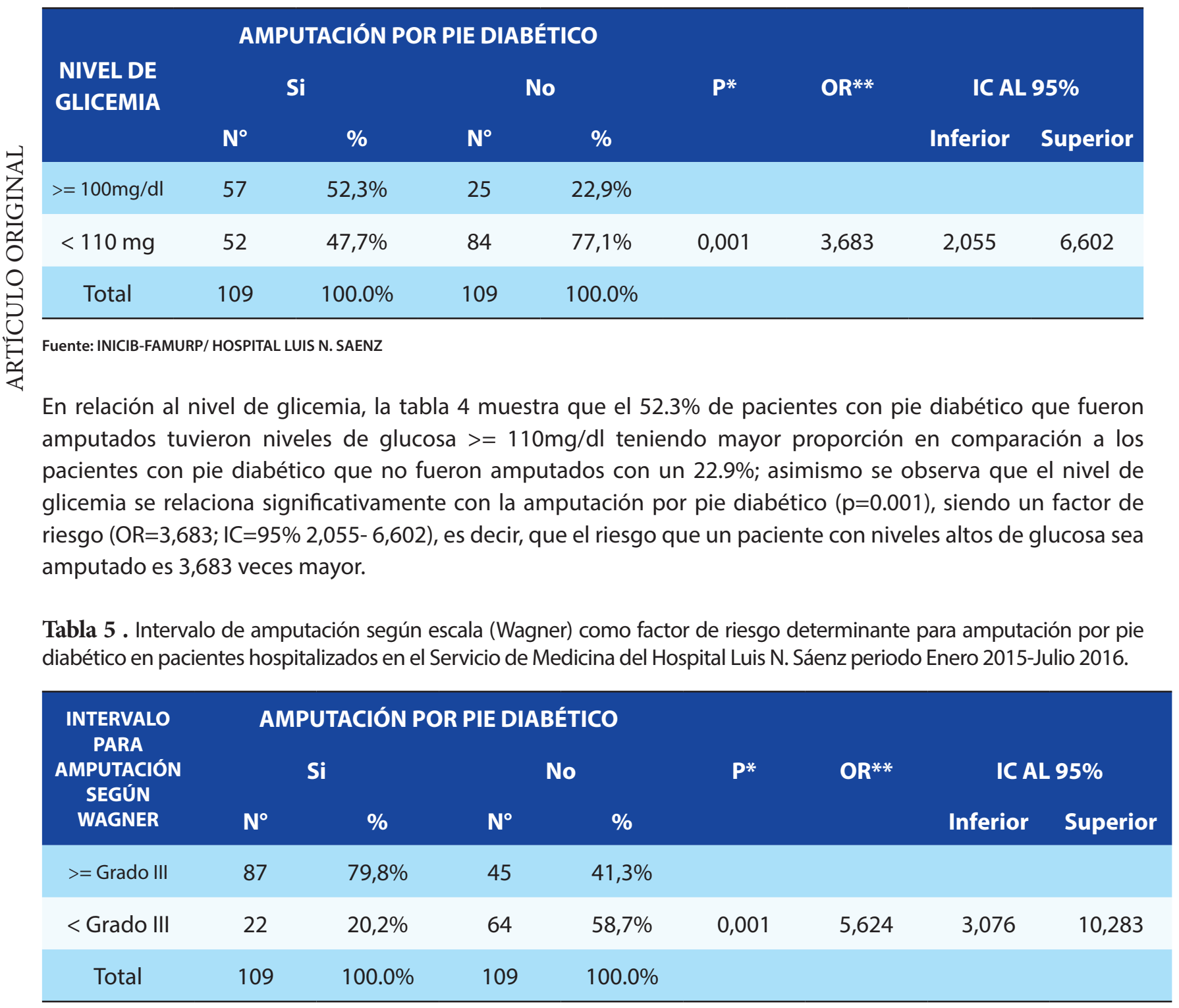

Fuente: INICIB-FAMURP/ HOSPITAL LUIS N. SAENZ

Respecto a la tabla 5, se observa que el 79.8\% de pacientes con pie diabético que tuvieron un grado de lesión Wagner mayor e igual a III fueron amputados teniendo mayor proporción en comparación con pacientes que presentaron un grado menor a III según la escala de Wagner con un $20.2 \%$, se observa que a un grado mayor e igual a III según la escala de Wagner se relaciona significativamente con la amputación por pie diabético ( $p=0,001)$, siendo un factor de riesgo para la amputación de pie diabético $(O R=5,624 ; I C=3,076-10,283)$; es decir el riesgo que un paciente con pie diabética Wagner mayor e igual a III sea amputado es 5,624 veces mayor que un paciente con Wagner menor a III. 


\section{DISCUSIÓN}

Al realizar el análisis de los datos obtenidos en relación a los factores de riesgo determinantes para la amputación de pacientes con pie diabético se encontró: En cuanto a la edad se halló en nuestro estudio que los pacientes con edad mayor o igual a 75 años presentan mayor riesgo de amputación por pie diabético $O R=3,808 ;(I C=2,103-6,897)$, resultado que se encuentra respaldado con un estudio realizado por Mayque Guzmán Cayado, et al ${ }^{14}$ donde obtuvieron que la edad en donde se presentó el mayor número de amputaciones fue de 77 años vs 76 años tanto para el sexo femenino como el sexo masculino respectivamente. Se ha visto en diferentes estudios que a mayor edad por lo general hay mayor tiempo de evolución de la enfermedad y esto aumenta el riesgo de amputaciones por pie diabético. Por otra parte hay un estudio realizado por Paola Cieza Zulueta ${ }^{16}$ en donde reporta, que en la edad mayor a 76 años, predominaron las amputaciones en pacientes con pie diabético, con lo cual apoyamos nuestro resultado encontrado en la población con pie diabético. Se encontró en la población estudiada que el sexo masculino se relaciona significativamente con la amputación $(p=0,011)$. Considerándose factor de riesgo para amputación por pie diabético $(\mathrm{OR}=2,460$; $\mathrm{IC}=1,217-4,972)$; es decir que el riesgo de ser de sexo masculino es 2,46 veces mayor que las pacientes de sexo femenino para ser amputadas por presentar dicha alteración. En un estudio publicado por Zubair et $\mathrm{al}^{8}$ nos indica que el sexo masculino también es un factor de riesgo que conlleva a la amputación por pie diabético con un $\mathrm{OR}^{2,8}$, lo que apoya nuestro resultado encontrado en el presente estudio. Existe un estudio nacional de Nathali Leiva Cañarín ${ }^{12}$ que hace referencia que el sexo masculino predomina sobre el femenino en un $71.2 \%$ siendo un valor elevado al igual que en nuestro estudio con respecto con un $87.2 \%$ de pacientes de sexo masculino con mayor riesgo de amputación. Según los estudios de la National Hospital Discharge Survey (NHDS) ${ }^{34}$ la tasa de amputaciones entre hombres es superior a la reportada en mujeres siendo demostrado en estudios epidemiológicos, pero no se ha podido explicar claramente cuál sería la causa que desencadena mayor número de amputaciones en pacientes varones. El tiempo de evolución de la Diabetes Mellitus mayor/igual a 10 años resultó ser un factor de riesgo para amputación en pacientes con pie diabético, se observó que se relaciona de manera significativa con la amputación ( $\mathrm{p}=0.001)$, siendo un factor de riesgo $(\mathrm{OR}=3,206 ; \mathrm{IC}=95 \% 1,578-6,514)$ lo que quiere decir que el riesgo de amputación de un paciente con pie diabético es 3,206 veces mayor que en quienes presentan la enfermedad por menos de 10 años. En estudios previos se han observado resultados similares como en el estudio de Arana Bardales Carlos Eduardo ${ }^{8}$ donde se obtuvo como resultado el tiempo de enfermedad de diabetes mellitus mayor a 10 años (OR=2,14 IC=95\% 1,417-4,121) concluyendo así que es un factor de riesgo para la amputación. Otro estudio realizado por Bach Rodrigo Jesús Flores Palacios en relación a la variable estudiada nos muestra que el tiempo de enfermedad de 10 años o más tuvo un $\mathrm{OR}=3,67$ (IC95\% 1,79 - 7,45) con $\mathrm{p}=0,00$ lo cual nos indica que tanto en nuestro estudio como en el antecedente existe significancia entre el mayor tiempo de enfermedad y la amputación de pie diabético. A pesar de encontrar estudios que nos respaldan existen un estudio por Calderón $\mathrm{M}$ y Giovanna $\mathrm{F}$ en donde los pacientes de la muestra en estudio con un tiempo de evolución de la Diabetes Mellitus tipo $2>10$ años presentan un $\mathrm{OR}=0.78$ indicándonos de esta manera que existe asociación negativa por lo cual se considera un factor protector el mismo que no tiene significancia estadística por presentar valor $p=0.25$. Dentro del factor de riesgo que involucra el nivel de glicemia $>=110 \mathrm{mg} / \mathrm{dl}$ tenemos una relación significativamente con la amputación por pie diabético $(p=0,01)$, siendo un factor de riesgo para la amputación $(\mathrm{OR}=3,683$; IC $=95 \% 2,055-6,602)$ por lo que el riesgo de un paciente con pie diabético con niveles altos de glucosa sea amputado es 3,683 veces mayor; así mismo el estudio de Zubair ${ }^{8}$ apoya nuestro resultado al encontrar que el nivel elevado de glicemia mayor a $120 \mathrm{mg} / \mathrm{dl}$ es un factor de riesgo $(\mathrm{OR}=5,44)$ siendo en este caso el riesgo de 5,4 veces mayor para la amputación de pie diabético. Los valores de glicemia considerados en nuestro estudio fue >= $110 \mathrm{mg} /$ dl establecido como límite superior en nuestro campo de estudio "Hospital Luis N. Sáenz", de este modo existen estudios de Análida Elizabeth Pinilla, María del Pilar Barrera, Cristhian Rubio, Diego Devia ${ }^{13}$ sobre actividades de prevención y factores de riesgo en diabetes mellitus y pie diabético en donde indican finalmente en su estudio que uno de los factores para riesgo de amputación es la hiperglicemia.

Durante el estudio realizado en el Hospital Luis N. Sáenz periodo Enero 2015 - Julio 2016 indicó que el grado de lesión Wagner mayor e igual a III es una variable significativa con relación a la amputación ( $p=0.001)$ considerada a su vez como un factor de riesgo para la amputación de pie diabético $(\mathrm{OR}=5,624$; IC=3,076-10,283); es decir que un paciente con pie diabético Wagner mayor e igual a III tiene riesgo de 
amputación de 5,624 veces más que un paciente con Wagner menor a III. Gabriel Vidal Domínguez ${ }^{2}$ en su estudio nos indica que el grado de lesión Wagner IV se presenta como factor de riesgo para amputación por pie diabético $(\mathrm{OR}=8,0$; IC95\%=3,12-20,53), el cual estarías apoyando nuestro estudio por encontrarse sobre el intervalo planteado. Otro estudio que respalda nuestro resultado es el realizado por Paola Cieza Zulueta $^{16}$, reporta que según el estadiaje de Wagner, el grado $\mathrm{V}$ el más frecuente (73.47\%); igualmente en el Hospital Nacional Arzobispo Loayza Nathali Leiva Cañarí12 encontró una mayor frecuencia de grado IV según la clasificación de Wagner en el $52.9 \%$ de los casos, ambos estudios demuestran que el grado de lesión mayor e igual a III según Wagner son factores de riesgo para amputación.

\section{REFERENCIAS BIBLIOGRÁFICAS}

1. David E, Leonid L, Julio G, Giannina E: Amputación del Miembro Inferior por Pie Diabético en Hospitales de la Costa Norte Peruana 1990 - 2000 Características Clínico-Epidemiológicas. Rev. Perú med exp salud publica 2003; 20 (3): $138-139$

2. Gabriel Vidal-Domínguez: Factores de riesgo de amputación en el pie diabético. Rev. Soc. Perú Med Interna 2010; vol. 23 (4): 146.

3. Gamarra C. Características clínicas y factores para pie diabético en pacientes que acuden a la unidad de pie diabético del Hospital Nacional Dos de Mayo. (Internet). Lima: 2003. (Consultado Jun 2009). Disponible en: http:/sisbib.unmsm.edu.pe/bibivrtual/mon ografíassalud/gamarra_cm/ contenido.htm

4. Rodrigo F. Factores Asociados al Desarrollo de Pie Diabético en Pacientes con Diabetes Mellitus tipo 2 en el Servicio de Medicina Interna 03 y Pie Diabético del Hospital Nacional Guillermo Almenara Irigoyen durante el año 2012. (1 - 2).

5. Harold T, Cesar G, Jaime P, Rosa P y Hugo A: Características clínicas y epidemiológicas de los pacientes hospitalizados por pie diabético en el Hospital Nacional Dos de Mayo entre 2006 y 2008, Lima-Perú. Rev. Perú. Epidemiol. Vol. 16 Nro. 3 Diciembre 2012.

6. Jordi R. Pie diabético, Abordaje multidisciplinar. FMC. 2015;22(5):242

7. Dr. Mayque G, Dra. Tamara B, Dr. Manuel C y Dr. Juan P. Algunos Factores Clínicos de Riesgo de Amputación en un grupo de Diabéticos del Municipio Artemisa. Rev. Cubana Invest Biomed 2006; 25(1): 5 - 6 .

8. Arana B. Factores Predictivos de Amputación en Pacientes con Pie Diabético. (Internet). Lima: 2015. Disponible en: http://repositorio.upao. edu.pe/bitstream/upaorep/1306/1/ARANA_CARLOS_FACTORES PREDICTIVOS_AMPUTACI\%C3\%93N.pdf.

9. Álvarez J., Carreño J., Rodríguez J. Amputaciones en el Pie Diabético. Capitulo X.

10. J.M. Fernández y J.A. Tirado: Repercusión socioeconómica de las amputaciones en el pie diabético. Angiología. 2013;65(Esp Cong):59-62

11. Aguilar M y Gonzales R: Amputación de Miembro Inferior por Pie Diabético en Pacientes con Diabetes Tipo 2, en el Hospital San Juan de Dios de Santa Ana, Periodo 2007-2009. (Internet). Disponible en: http://ri.ues. edu.sv/832/1/10136794.pdf.

\section{CONCLUSIÓN}

En nuestro estudio encontramos que los factores de riesgo determinantes para la amputación de pacientes con pie diabético hospitalizados en el Servicio de Medicina del Hospital Luis N. Sáenz, fueron: la edad mayor/igual a 75 años, el sexo masculino, el tiempo de evolución mayor/igual a 10 años, el nivel de glicemia mayor/igual a $110 \mathrm{mg} / \mathrm{dl}$ y el grado de lesión mayor/ igual a III según la escala de Wagner.

Financiamiento: Autofinanciado.

Conflicto de interés: Los autores declaran no tener conflictos de interés en la publicación de este artículo.

Recibido: 21 de marzo del 2017

Aprobado: 30 de marzo del 2017
12. Nathali L. Factores clínicos de riesgo de amputación en pacientes diabéticos hospitalizados en el servicio de medicina del Hospital Nacional Arzobispo Loayza en el año 2015. (Internet). Disponible en: http://cybertesis. unmsm.edu.pe/bitstream/cybertesis/4711/1/Leiva_cn.pdf

13. Analida P, María del Pilar B, Nelcy R, Cristhian R y Diego D. Prevalencia de las Actividades de Prevención del Pie Diabético y de los Factores de Riesgo Asociados en Pacientes Diabéticos Hospitalizados en la Clínica Universitaria Carlos Lleras Restrepo. (Internet). Disponible en: http://www.bdigital.unal. edu.co/7163/1/597763.2011.pdf.

14. Mayque G, Tamara B, Manuel C y Juan P: Algunos factores clínicos de riesgo de amputación en un grupo de diabéticos del municipio Artemisa. Rev Cubana Invest Biomed 2006;251

15. Aguilar M, Walter A, Gonzales R y Jaime A: Amputación de Miembro Inferior por Pie Diabético en Pacientes con Diabetes Tipo 2, en el Hospital San Juan de Dios de Santa Ana en el periodo 2007 a 2009.

16. Paola C. Prevalencia de Pie Diabético y Riesgo de Amputación de Miembro Inferior en Pacientes del Hospital Manuel Ignacio Montero Periodo 2000-2008.

17. Sereday M, Damiano M, Lapertosa S, Cagide A y Bragagnolo JC. Amputaciones de Miembros Inferiores en Diabéticos y No Diabéticos en el ámbito hospitalario. Asociación Latinoamericana de Diabetes.

18. Jose B, Jose F, Armando H, Nelson Ch y Manuel H. Caracterización del pie diabético y algunos de sus factores de riesgo. Rev. Cubana Angiol y Cir Vasc 2010; 11(1): 10-18

19. Valentina C y Ana Paula G. Factores de Riesgo para Pie Diabético en Pacientes con Diabetes Mellitus Tipo 2.

20. Yamilet E, Ubaldo T, Odalys P, Neyda F, Vladimir I y Eva R. El Heberprot en el tratamiento de úlceras del pie diabético. Biotecnol Apl. 2010; 27(2):151-4.

21. Yudit G, Jose A, Barnes D, Daniel G y Adrian E. Primera amputación de miembros inferiores en pacientes diabéticos. Memorias Convención Internacional de Salud Pública. Cuba Salud 2012. La Habana 3 -7 de diciembre de 2012.

22. Guillermo P. Amputaciones en un Hospital General en Pacientes con Pie Diabético. Análisis de tres años. Revista salud quintana roo - issn 2007 - 1671. 
23. Analida P, María del Pilar B, Cristhian Ry Diego D. Actividad de prevención y factores de riesgo en diabetes mellitus y pie diabético. Acta Medica Colombiana Vol. 39 №3- Julio-Setiembre 2014.

24. Caleb A y Sandy B. Estudio descriptivo transversal realizado en los Servicios de Cirugía de Hombres y Mujeres del Hospital Roosevelt y Cirugía de Emergencia del Instituto Guatemalteco de Seguridad Social de Zona 9 agostoseptiembre del 2014.

25. Harold L, Cesar G, Jaime P, Rosa P y Hugo A. Características clínicas y epidemiológicas de los pacientes hospitalizados por pie diabético en el Hospital Nacional Dos de Mayo entre 2006 y 2008, Lima-Perú. REV. PERU. EPIDEMIOL. VOL 16 NO 3 DICIEMBRE 2012.

26. Organización Mundial de la Salud. Diabetes. Nota descriptiva №312. Enero de 2015.

27. Escuela de medicina. Diabetes Mellitus: Definición y Etiopatogenia. (Internet). Disponible en: http://escuela.med.puc.cl/paginas/cursos/ tercero/IntegradoTercero/ApFisiopSist/nu tricion/NutricionPDF/ DiabetesMellitus.pdf

28. Luis N, José S, Oscar C y Freddy G. Pie Diabético. Rev. Soc. Perú Med Interna 2012; vol. 25 (2) 77.

29. Rosa C, Juan F y Francisco C. Guía de Práctica Clínica en el pie diabético. Archivos de Medicina. Vol. 10 No. 2:1 doi: 10.3823/1211. Disponible en: www. archivosdemedicina.com

30. Analida P, Maria B, Ana S, Arturo M. Factores de riesgo en diabetes mellitus y pie diabético: un enfoque hacia la prevención primaria. Revisión de temas-Cardiologia del adulto, Bogota Colombia (Internet) 2013.
31. OMS. Día Mundial de la Diabetes: muchas de las amputaciones que acarrea la enfermedad se podrían evitar (Internet). Disponible en: http:// www.who.int/mediacentre/news/releases/2005/pr61/es/

32. LeMaster J, Mueller M, Reiber G, Mehr D, Madsen R, Conn V. Effect of weightbearing activity on foot ulcer incidence in people with diabetic peripheral neuropathy.American physical therapy association.2008;88:1385-136

33. Lobmann R, Kayser R, Kasten G, Kasten U, Kluge K, Neumann W, et al. Effect of preventative footwear on foot pressure as determined by pedobarography in diabetic patient: a prospective study. Diabet. Med. 2001; 18:314-319

34. Calderon G. Tiempo de evolución de diabetes mellitus tipo 2 como factor predisponente de amputación en pacientes con pie diabético en el Hospital Victor lazarte Echegaray (Internet). Trujillo: 2014. Disponible en: http://repositorio.upao.edu.pe/bitstream/u paorep/430/1/CALDERON_ GIOVANNA_DIABETES_MELLITUS_AMPUTACION.pdf

35. CDC. National Center for Health Statistics (Internet). 2014-2015. Disponible en: https://www.cdc.gov/nchs/index.htm

36. Flores B. Factores Asociados al Desarrollo de Pie Diabético en Pacientes con Diabetes Mellitus Tipo 2 en el Servicio de Mediana Interna y Pie Diabético del Hospital Nacional Guillermo Almenar Irigoyen durante el Año 2012 (Internet). Disponible en: http://200.37.105.196:8080/bitstream/ han dle/unjbg/197/82_2013_Flores_Palacios_RJ_FACS_Medicina_2013_ Resumen.pdf?sequence $=2$

\section{Indizado en: latindex}

http://www.latindex.org/latindex/ficha?folio=14280

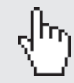

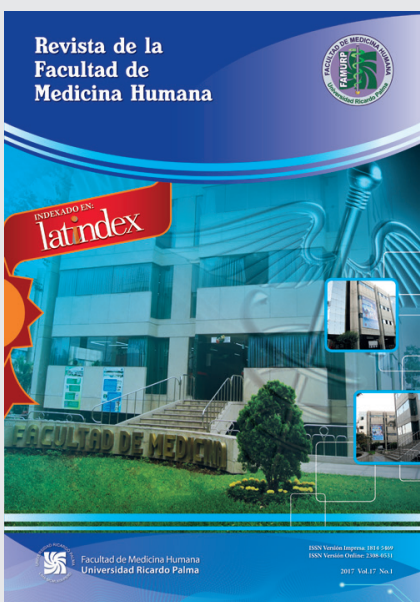

